\title{
Terahertz NDE for Metallic Surface Roughness Evaluation
}

\author{
Robert F. Anastasi ${ }^{1 *}$ and Eric I. Madaras ${ }^{2}$ \\ ${ }^{1}$ U.S. Army Research Laboratory, Vehicle Technology Directorate, AMSRD-ARL-VT-SM, \\ Nondestructive Evaluation Sciences Branch, NASA Langley Research Center, Hampton, VA 23681 \\ ${ }^{2}$ NASA Langley Research Center, Nondestructive Evaluation Sciences Branch, \\ Hampton, VA 23681
}

\begin{abstract}
Metallic surface roughness in a nominally smooth surface is a potential indication of material degradation or damage. When the surface is coated or covered with an opaque dielectric material, such as paint or insulation, then inspecting for surface changes becomes almost impossible. Terahertz NDE is a method capable of penetrating the coating and inspecting the metallic surface. The terahertz frequency regime is between $100 \mathrm{GHz}$ and $10 \mathrm{THz}$ and has a free space wavelength of 300 micrometers at $1 \mathrm{THz}$. Pulsed terahertz radiation, can be generated and detected using optical excitation of biased semiconductors with femtosecond laser pulses. The resulting time domain signal is 320 picoseconds in duration. In this application, samples are inspected with a commercial terahertz NDE system that scans the sample and generates a set of time-domain signals that are a function of the backscatter from the metallic surface. Post processing is then performed in the time and frequency domains to generate $\mathrm{C}$-scan type images that show scattering effects due to surface non-uniformity.
\end{abstract}

Keywords: Terahertz, Nondestructive Evaluation, Surface Roughness

\section{INTRODUCTION}

Roughness is a measure of the relative elevations of surface irregularities that result from various sources such as machining processes, surface damage, and corrosion. In machining applications, a machinist will compare his work piece to a representative sample or standard by running his finger over the work piece and sample. The drawback of this method is that it is qualitative with different machinists arriving at different conclusions for the same work piece. A contact surface-tracing machine is a quantitative method used to measure roughness that involves pulling a stylus across the surface. Non-contact optical methods can measure the scattering of radiation by the surface being characterized and yield statistical surface parameters [1,2]. One instrument for non-contact surface roughness measurement is a Total Integrated Scattering (TIS) instrument. This instrument measures the specular and diffusely scattered laser light from a surface and relates these measurements to the root-mean-squared (rms) surface roughness. This general relationship is [2]

$$
T I S=\frac{R_{s}}{R_{0}}=e^{-(4 \pi \delta / \lambda)^{2}}
$$

where $R_{s}$ is the specular reflectance, $R_{0}$ is the total of the specular reflection $\left(R_{s}\right)$ and the diffuse reflectance $\left(R_{d}\right), \lambda$ is the wavelength of light, and $\delta$ is the rms surface roughness. In this relation, it is assumed that the light is incident normal to the surface. This non-contact method requires direct view of the surface being interrogated. If the surface is covered by paint, foam or other dielectric nontransparent material then direct interrogation is not possible. Terahertz

* Correspondence: email: robert.f.anastasi@nasa.gov; phone: 757-864-3391; fax: 757-864-4914 
(THz) nondestructive evaluation (NDE) can potentially evaluate subsurface metallic materials because $\mathrm{THz}$ radiation can penetrate a dielectric material and inspect an underlying metallic surface. THz NDE is relatively new inspection technology $[3,4]$ that offers a non-contact and high-resolution inspection method. Its frequency regime is between 100 $\mathrm{GHz}$ and $10 \mathrm{THz}$ and it has a free space wavelength of $300 \mu \mathrm{m}$ at a frequency of $1.0 \mathrm{THz}$. This radiation, like microwaves, is able to penetrate and inspect nonconductive material for defects and is reflected from metallic materials, but pulsed $\mathrm{THz}$ NDE returns an electromagnetic time response signal that can be interpreted in a manner comparable to conventional ultrasonic time-domain signals as opposed to the continuous wave methods used in microwave NDE. Some other uses of $\mathrm{THz}$ have focused on spectroscopic applications such as inspecting the chemical content of packages [5], inspection of artwork and diagnostics of skin burn depth and severity [6], photonic crystal characterization [7], and response of $\mathrm{THz}$ to human skin [8]. In this paper, the response of a THz NDE system is examined as a function of surface roughness.

\section{SETUP AND SAMPLES}

The THz system used in this work is manufactured by Picometrix, Inc and is similar to systems designed to inspect up to $30 \mathrm{~cm}$ of sprayed-on-foam insulation on the Space Shuttle external tank [9]. For this system, $\mathrm{THz}$ signals are generated and detected, using optical excitation of biased semiconductors with 70-femto-second pulses from an $800 \mathrm{~nm}$ laser. The resulting digitized time-domain signal is composed of 2048 points and has a duration of $320 \mathrm{ps,} \mathrm{which}$ corresponds to a propagation path length in air of $96 \mathrm{~mm}$ or approximately 4 inches. The transmitter and receiver units were in a confocal arrangement and use a $15 \mathrm{~cm}$ diameter, $285 \mathrm{~mm}$ focal length aspherical lens to concentrate the $\mathrm{THz}$ energy on the sample. The spot size diameter at the focus is approximately $3 \mathrm{~mm}$. This transceiver head is scanned in a raster pattern over the sample while time-domain signals are digitized and stored for later processing. In the present work, no modifications were made to the transceiver other then scan height adjustments. The transceiver arrangement is shown in figure 1a and a sample $\mathrm{THz}$ signal reflected from an aluminum plate and its spectrum is shown in figure $1 \mathrm{~b}$. The beam spread of the system was measured by placing a variable aperture between the THz-transmitter and focusing lens while monitoring the output signal amplitude. The full angle beam spread was approximately $27^{\circ}$ and the beam diameter at the focusing lens was approximately $9.5 \mathrm{~cm}$, thus the system f/number is approximately 3 and the Rayleigh limit of resolution is $\sim 1 \mathrm{~mm}$ at a frequency of $1 \mathrm{THz}$.

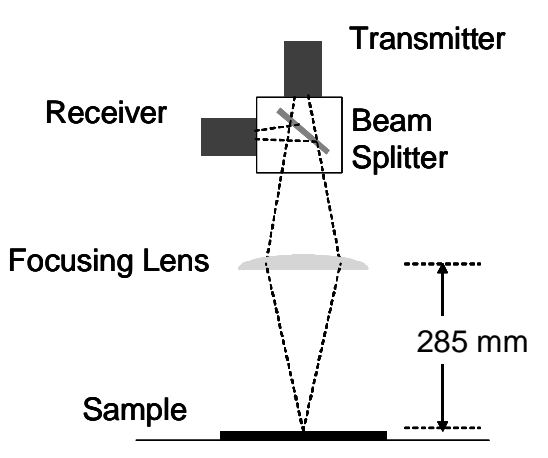

(a)
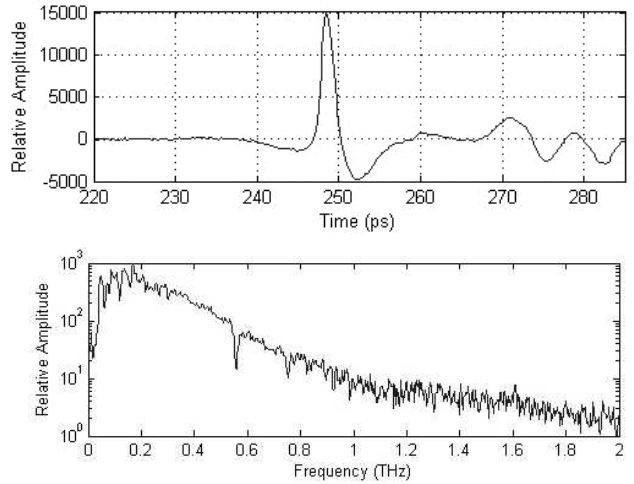

(b)

Figure 1. (a) Transceiver schematic and (b) a sample THz signal reflected from an aluminum plate and its spectrum. 
(a)

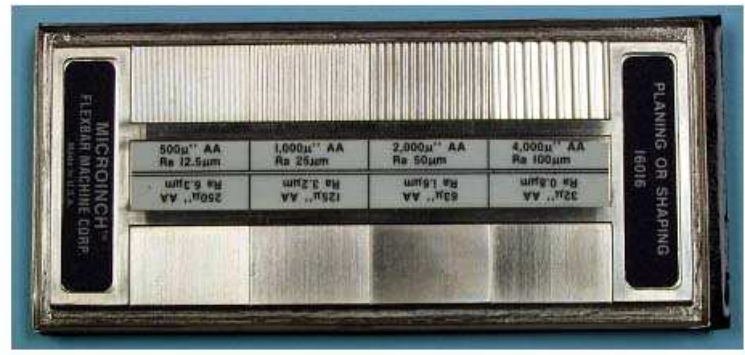

(b)

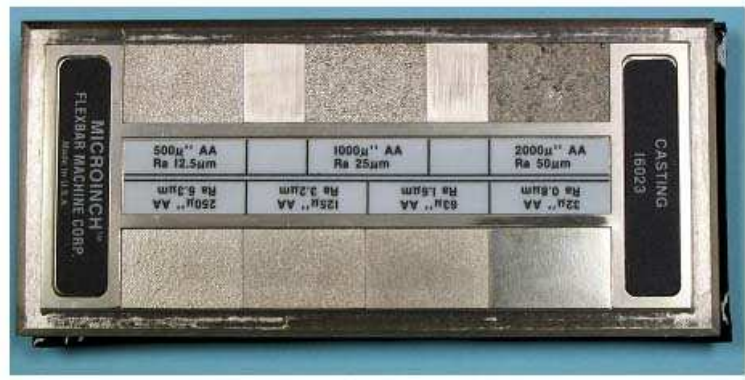

Figure 2. The surface roughness comparator plates (a) 'Planing or Shaping' plate and (b) 'Casting' plate

The samples used for this work were comparator plates made of electroformed nickel and are standards that a machinist would use to evaluate his work. Two different comparator plates were used: a 'Planing and Shaping' plate and a 'Casting' plate. The overall dimension of each plate was $5.5 \mathrm{~cm} \mathrm{x} 12.5 \mathrm{~cm}$ and each roughness area was approximately $16 \mathrm{~mm} \times 25 \mathrm{~mm}$. These plates covered a range of average roughness (Ra) from $0.04 \mu \mathrm{m}$ to $100 \mu \mathrm{m}$, where the average roughness is defined as the area between the roughness profile and its mean line, or the integral of the absolute value of the roughness profile height over the evaluation length [10]. The plates are shown in figure 2. The 'Planing and Shaping' plate has eight separate areas of roughness. The smoothest area has an average roughness of $0.8 \mu \mathrm{m}$ and the roughest is $100 \mu \mathrm{m}$. The roughness areas on this plate appear as parallel rulings. For the $100 \mu \mathrm{m}, 50 \mu \mathrm{m}, 25 \mu \mathrm{m}$, and 12.5. $\mu \mathrm{m}$ areas the ruling spacing is $3.2 \mathrm{~mm}, 1.6 \mathrm{~mm}, 0.8 \mathrm{~mm}$ and $0.4 \mathrm{~mm}$ respectively. The 'Casting' plate has seven separate areas of roughness that have a range of average roughness from $50 . \mu \mathrm{m}$ to $0.8 . \mu \mathrm{m}$. These roughness areas appear as randomly rough surfaces. The average roughness parameter ( $\mathrm{Ra})$ has an approximate relation to the rms roughness $(\delta)$ used in equation (1), [1].

$$
R a \cong 0.8 \delta
$$

\section{OPTICAL RAY TRACING MODEL}

To gain a better understanding of how the THz system's optical configuration might influence roughness measurement, an optical ray-tracing model was built and its results were compared to the experimental measurements. In this simulation, the sample was modeled as a mirror with its surface modified using a function that adds surface roughness by symmetrically rotating a surface element about its normal. To estimate the rms roughness, it was assumed that the surface element was rotated about it centerline and a corresponding surface height could be calculated. A series of surface height values were obtained by randomly varying the angle. This series of values was then used to calculate an rms value for the prescribed angle and then used as the rms surface roughness estimate for the ray tracing mode simulations. Figure 3 shows three ray tracing results for rotations of $0^{\circ}, 10^{\circ}$, and $30^{\circ}$ or corresponding estimated rms values of $0 \mu \mathrm{m}, 96 \mu \mathrm{m}$, and $149 \mu \mathrm{m}$. This figure shows that as roughness increases, ray scattering increases. However, figure 3a shows some rays deviate from the predicted path. This appeared to be a function of the aspherical lens surface defined in the ray-tracing program because ray deviation does not occur when a convex-planar lens was used in place of the aspherical lens. To overcome this issue a large number of rays were used to average out any ray tracing errors. To quantify the modeling results the number of rays at the receiver was used as a measure of received specular signal intensity. 


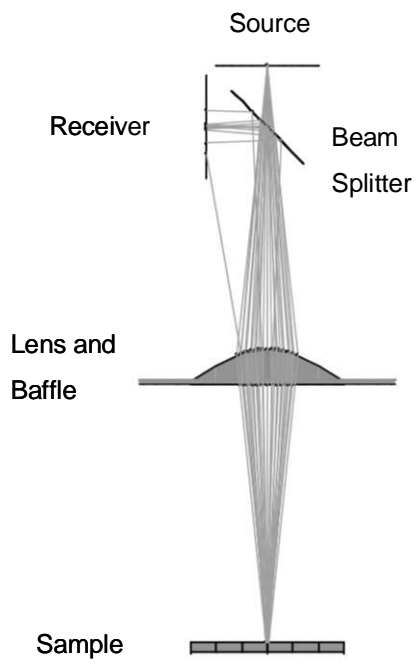

(a)

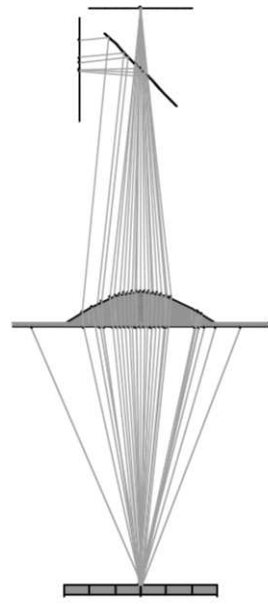

(b)

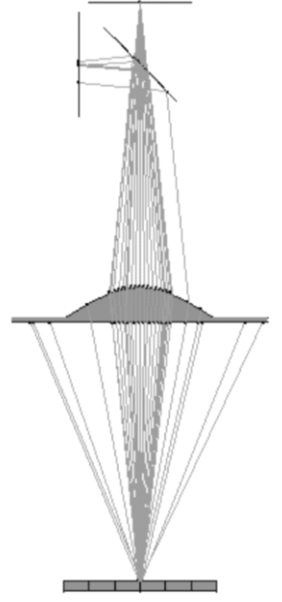

(c)

Figure 3. Ray tracing model examples with estimated surface roughness of (a) $0 \mu \mathrm{m}$, (b) $96 \mu \mathrm{m}$, and (c) $149 \mu \mathrm{m}$

(1)

(2)

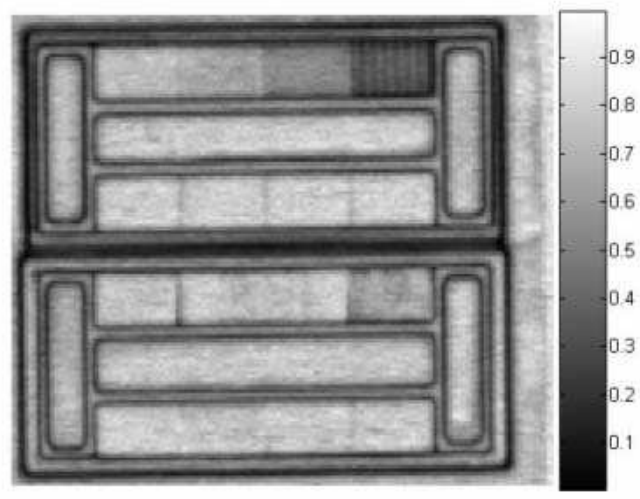

(a)

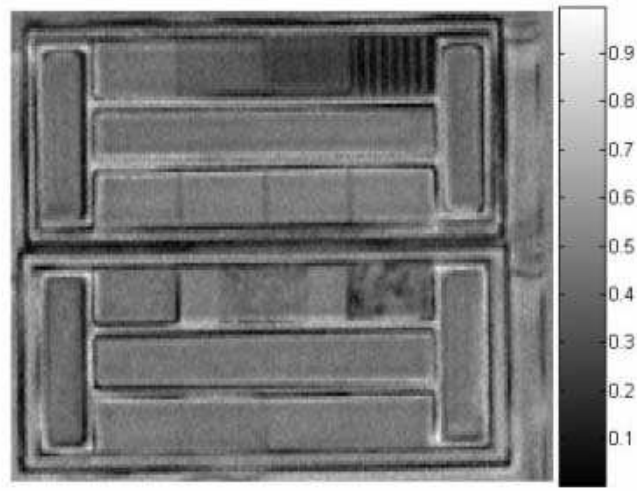

(b)

Figure 4. The (a) amplitude and (b) frequency images for Plate 1: 'Planing and Shaping' plate and Plate 2: 'Casting' plate.

\section{MEASUREMENTS AND RESULTS}

The samples were inspected with the THz system and the scan data was stored for post processing. To visualize the data, amplitude and frequency images were generated. The amplitude image was generated by plotting the maximum amplitude of each signal as a function of location and the frequency image was generated by calculating the power spectrum of each signal in the THz scan data and then taking the mean amplitude of the spectrum about $0.4 \mathrm{THz}$. The image at this frequency exhibited the best contrast between the different roughness areas. Figure 4 shows the roughness areas in shades of gray. The darker gray areas correspond to higher roughness and the lighter shades of gray correspond to less roughness. Surface roughness below $12.5 \mu \mathrm{m}$ Ra became indistinguishable from other surfaces. 
To quantify the scan data, maximum signal amplitude and spectral content for each roughness area on the comparator plates were examined. The spectral content of the signals in each roughness area was examined by calculating the average power spectrum of all the signals in a roughness area. Figure 5 shows the average power spectrum for each roughness area on the 'Planing and Shaping' plate. The spectral differences can be identified for the $12.5 \mu \mathrm{m}, 25 \mu \mathrm{m}, 50$ $\mu \mathrm{m}$, and $100 \mu \mathrm{m}$ roughness between 0.3 and $0.6 \mathrm{THz}$. For the smoother areas, the spectra are similar and overlap. This illustrates that the measurement was bandwidth limited. Note that the frequency minimum at about $0.55 \mathrm{THz}$ was a water vapor absorption line.

TIS measurements were obtained by taking the ratio between the specular and total reflection. The specular reflection for each roughness area was calculated by averaging all the signals in each roughness area and then taking its maximum amplitude as the total specular reflection. For the total reflection, the maximum amplitude of the smoothest roughness area was used, in practice a smooth standard maybe used. The standard deviation of this average per roughness area was used to estimate $\mathrm{a} \pm 0.03$ TIS error. A plot of this TIS value as a function of roughness for each of the comparator plates is shown in figure 6 along with calculated TIS values from equation (1) and TIS values calculated from the ray tracing model simulations. In the model simulations and calculations, a frequency of $0.4 \mathrm{THz}$ or wavelength of $0.75 \mathrm{~mm}$ in free space was used since this was approximately the center of the band-limited measurement.

The measurements, calculations, and modeling results in figure 6 show that as roughness increases the TIS value decreases and that the results diverge as roughness increases. The divergence may in part be due to geometry difference of the samples, where the 'Planing and Shaping' plate has a linear roughness and the 'Casting' plate has a random roughness. In addition, errors may be introduced because a TIS instrument is typically used to measures the diffuse reflectance at a single wavelength over a hemisphere while the $\mathrm{THz}$ system measures the specular reflectance over a range of frequencies and over a limited angle.

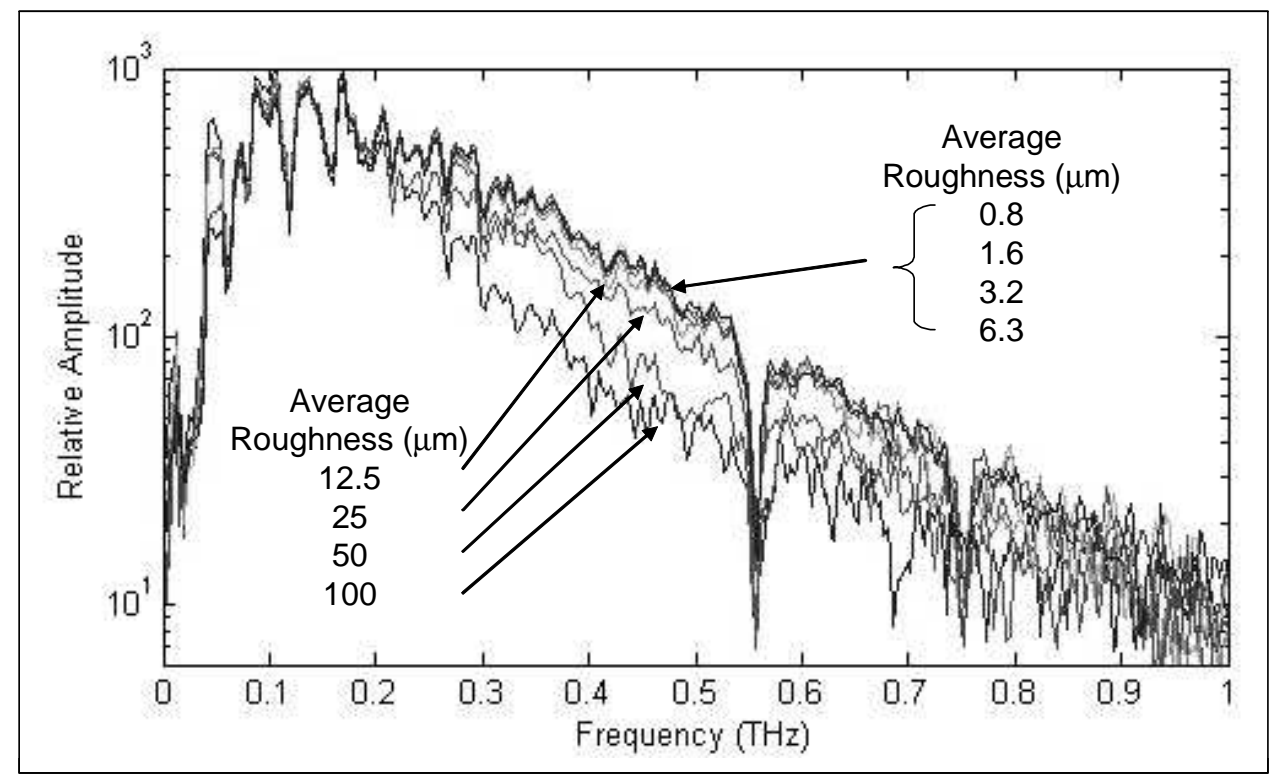

Figure 5. Average power spectrum for each roughness area on the 'Planing and Shaping' plate. 


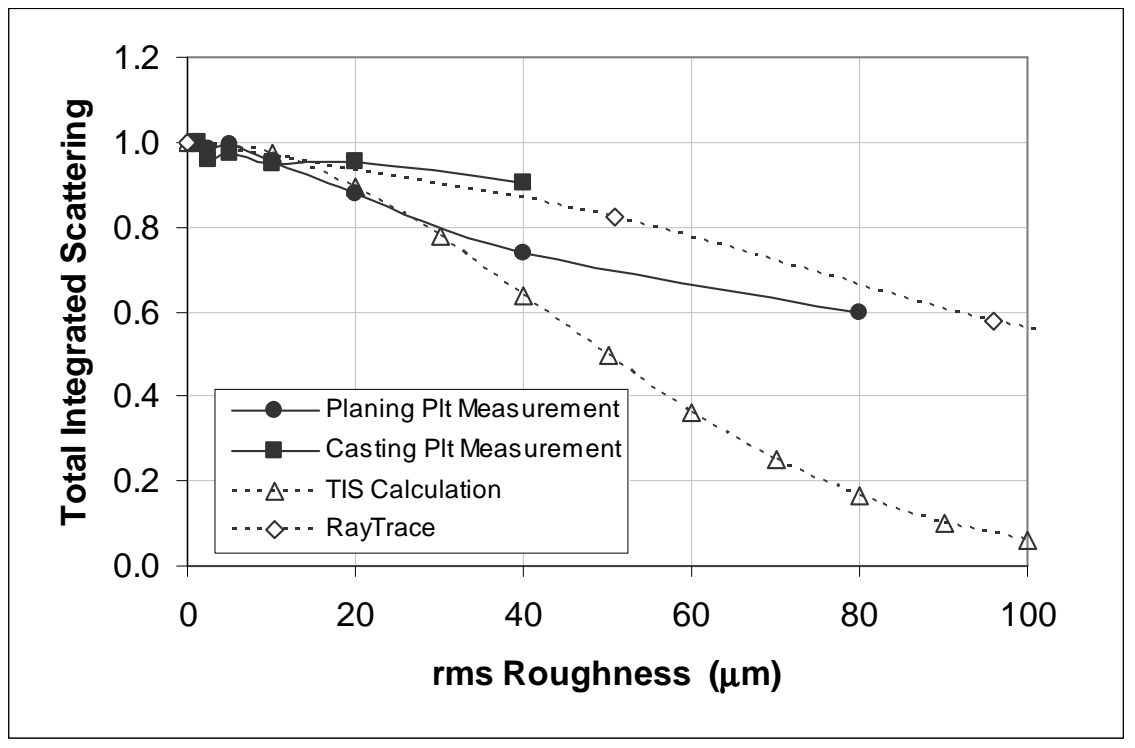

Figure 6. Measurement results as a function of rms roughness compared to calculated total integrated scattering values and ray tracing model results at a wavelength of $0.75 \mathrm{~mm}$ or frequency of $0.4 \mathrm{THz}$

\section{CONCLUSIONS}

Imaging of metallic surface roughness using THz NDE can be accomplished in a manner similar to conventional ultrasonic imaging. This paper shows that to quantify the data, methods of Total Integrated Scattering are plausible to adopt with the noted difference being a diffuse measuring system versus a specular measuring system, the THZ system and a single versus a band of wavelengths. The THz response to the rough surfaces showed that as roughness increased the scatter increased and thus the amplitude of the detected signal decreased. This can be seen in the images and the TIS versus Roughness plot where surface roughness below $12.5 \mu \mathrm{m}$ Ra or $\sim 10 \mu \mathrm{m}$ rms became indistinguishable from other surfaces. The best correspondence between measurements, formula, and modeling appear to be in the rms range from $\sim 10 \mu \mathrm{m}$ to $\sim 40 \mu \mathrm{m}$, above this range, the results diverge. This divergence may be due to instrument, modeling, and sample geometry differences. With modeling and technique refinements, the useful roughness measurement range may be extended.

\section{REFERENCES}

1. Ogilvy, J.A., "Theory of Wave Scattering from Random Rough Surfaces," Institute of Physics Publishing, Philadelphia, PA 1992

2. Bennett, J.M., and Mattsson, L. "Introduction to Surface Roughness and Scattering," Op Soc of America, Washington, D.C., 1999

3. Mittleman, D., et al., “T-Ray Tomography,” Optics Letters, Vol. 22, No. 12, June 1997.

4. Dorney, T., et al., "Imaging with Terahertz Pulses," International Symposium on Optical Science and Technology, July 2000.

5. Hu, B. and Nuss, M.C., "Imaging with Terahertz Waves," Optics Letters, Vol. 20, No. 16, 1995.

6. Mittlemann, D.M., "Recent Advances in Terahertz Imaging," Applied Physics B, 68, 1999.

7. Nemec, H., et al., "Time-Domain Terahertz Study of Defect Formation in One-Dimensional Photonic Crystals," Applied Optics, Vol. 43, No. 9, 2004.

8. Pickwell, E., et al., "In Vivo Study of Skin Using Pulsed Terahertz Radiation," Physics in Medicine and Biology, 49, 2004.

9. Zimdars, D., Valdmanis, J. A., White, J. S., Stuk, G., Williamson, S., Winfree, W.P, and Madaras, E. I., "Technology and Applications of Terahertz Imaging Non-Destructive Examination: Inspection of Space Shuttle Sprayed On Foam Insulation," Review of Progress in Quantitative Nondestructive Evaluation, Golden, Colorado, 25-30 July 2004.

10. Precision Devices, Inc, http://www.predev.com/smg/parameters. htm\#Ra\%20-\%20Average\%20Roughness, November 2005. 